\title{
Research Article \\ Unequal Weighting for Improved Positioning in GPS-Less Sensor Networks
}

\author{
Thomas Haenselmann, Marcel Busse, Thomas King, and Wolfgang Effelsberg \\ Applied Computer Science IV, University of Mannheim, A5, 6-68159 Mannheim, Germany \\ Correspondence should be addressed to Thomas Haenselmann, haenselmann@informatik.uni-mannheim.de
}

Received 17 November 2006; Revised 9 July 2007; Accepted 8 October 2007

Recommended by Richard J. Barton

Positioning can be done by means of triangulation in which triples of position-aware nodes estimate a neighbor's position with the help of distances. If a larger number of nodes exist, multiple position estimates can be averaged to yield a more precise mean position. Rather than applying equal weights to each position estimate, we propose an unequal weighting scheme which emphasizes those summands in the averaging process that are more reliable than others. We will show that significant improvements of the overall estimate can be achieved, given that additional information about the error variance of a contributing estimate is known. A major advantage of the approach is that the precision of existing positioning systems can be improved without technical modifications.

Copyright (c) 2008 Thomas Haenselmann et al. This is an open access article distributed under the Creative Commons Attribution License, which permits unrestricted use, distribution, and reproduction in any medium, provided the original work is properly cited.

\section{INTRODUCTION}

The value of measured data in a sensor network increases significantly if each node's position is known, for example, to assign the measurement to a position on a map. However, the knowledge of the position of several nodes can also be helpful to jointly estimate an event's position like a distant explosion, a moving animal, or vehicle, which may not coincide with the coordinate of a particular node. Derivative information, like speed, acceleration, and so forth, can also be obtained by means of local or global coordinates [1]. Last but not least, some routing protocols require positioned nodes.

As a consequence, extensive research in recent years has focused on finding either global or local coordinates of nodes in a sensor network. A typical and still realistic assumption is that no global positioning system (GPS) is available. GPS is still not considered to be an option for sensor networks due to its price, form factor, and the energy consumption of the hardware available today. Besides, a line of sight between a sensor node and the satellites cannot always be ensured.

\subsection{Outline of our suggestion}

The novelty of our approach is that the presented model takes into account whether or not a location-aware node can con- tribute a more or less precise estimate of the position of a not yet positioned node. We will devise a weighting scheme that strengthens the influence of good estimates over bad ones.

In order to profit from our weighting approach, the following preconditions have to be met.

(i) The algorithm does not depend on the way distances are estimated. They can origin from measurements of the received signal strength as well as from time difference of arrival measurements or even from other kinds of estimates. However, it has to hold true that estimates of internode distances tend to become coarser on average with increasing distance. Note that in practice, a monotonic relationship between an increased distance and a decreased precision cannot be expected. However, our approach does not require such a monotonic behavior. We only need the tendency to get a larger error on average in the event of an increasing internode distance.

(ii) Antennas send roughly omnidirectional on average. This means, that the average antenna has no tendency to direct more power into a specific direction.

(iii) The measurements that lead to distance estimates should be calibrated in advance. In fact, this is no special assumption for this approach in particular but should be considered for all kinds of measurements. 


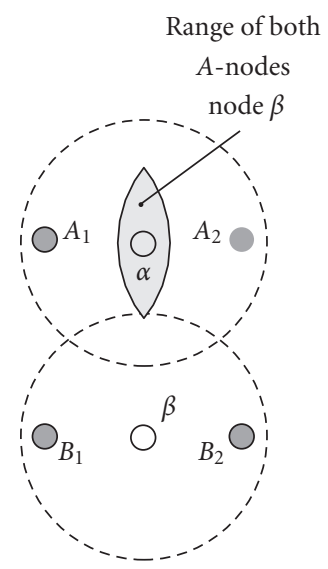

(a)

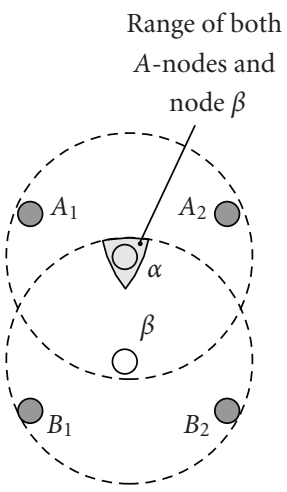

(b)
Figure 1: Localized nodes $A$ and $B$ are drawn darker than the unlocalized ones, $\alpha$ and $\beta$. On the left-hand side, $\alpha$ and $\beta$ are positioned based only on information about their localized neighbors. As a result, they are positioned right in the middle, respectively. With the additional information that $\alpha$ and $\beta$ are within a mutual radio range, the area of uncertainty (shown only for $\alpha$ on the right side of the figure) can be decreased significantly.

(iv) The positioning suggested aims at providing $2 \mathrm{D}$ coordinates only. But note that the convex combination used here works in the same way in $3 \mathrm{D}$, in particular as we use dimensionless expressions only.

Determining the distance measure itself is not the focus of this work, but we did some evaluations with the ESB sensor nodes (described in Section 4.1) on the suitability of radio and sound beacons for distance measurements.

\section{PREVIOUS WORK}

\subsection{Global positioning}

Approaches to the global positioning of a node need at least some nodes which have already acquired their position in world-coordinates.

The position-aware units may be a small number of advanced nodes which can be equipped with positioning systems to serve a much greater number of simple devices. After all nodes have been scattered in their destination area and once each has obtained its position, the failure of the advanced nodes would no longer be a problem. Similarly, a small number of simple nodes can be placed manually into the field of sensors. A human operator would have to obtain the exact global position, which could then be entered into the node before dropping it. Despite the simplicity of the approach, these manually initialized nodes could provide a location service for their fellows. The approach trades costs of nodes against effort for user interaction.

In order to obtain a global position for a node, an equally weighted average of the coordinates of all position-aware one-hop neighbors can be built according to an approach proposed by Bulusu et al. [2]. If the neighboring nodes form the vertices of a convex patch, then their average lies in the center of gravity of the patch. In a sense, this approach is in the spirit of the weighted convex combination proposed in this paper but with the additional difficulty that no mutual distance measures are available among nodes and that the quality of the radio or sound signal is not considered in the calculation of the position. We will, however, utilize this information in Section 3.

Another equally weighted sum of positions was proposed by Adebutu et al. [3]. The difference to the approach mentioned above is that from the beginning, both location-aware and unaware nodes are considered, as is shown in Figure 1. Obviously, position-unaware nodes are not helpful in the beginning. Initially, their position will be set to the position of an arbitrary neighbor or to that of another first guess. In each iteration, a node with a yet uncertain position builds the average coordinates of its neighbors, thus gaining certainty of its location. In the next iteration, its improved position will contribute to the calculation of the other nodes' positions, which will in the end converge against a stable result. The approach is a bit stronger than the previous one as it considers both positioned and not yet positioned nodes. Doherty et al. [4] adopted the same approach, choosing a linear programming scheme for the solution. All location-aware nodes are represented by constant locations, while nodes with uncertain positions are variable. The linear program has to be posed such that none of the existing one-hop connectivities will be violated.

\subsection{Relative positioning}

Another class of approaches requires no global coordinates. Nodes can localize themselves only with regard to their neighbors. In the beginning, local coordinates are computed only for the cluster of nodes within the same radio range. Later, those local coordinates are consolidated into an overall base for the entire network. The advantage of relative positioning is that nodes can estimate distances and angles among one another just as they would if using global coordinates. However, their location and orientation on the world map is unknown.

Čapkun et al. [5] have proposed such approach, which builds a local coordinate system (CS) if only mutual distances between nodes within the same proximity are known. The basic idea is that each node considers itself to be the origin of its own local CS. Without any global reference it has to define the direction into which its axes point. For the $X$-axis, this is done simply by choosing an arbitrary neighbor. By definition, the axis is declared to point towards that neighboring node. So far, this is a very abstract definition, because the neighbor is unlocalized as well.

The definition of the $Y$-axis is determined almost implicitly in 2D because it stands perpendicular to the $X$-axis. However, its direction can be flipped without violating the orthogonality constraint. Again, an arbitrary neighboring node is chosen and the direction of the $Y$-axis is declared to point towards that node. With the help of mutual distances, all remaining nodes in the proximity of the origin can now be localized within the artificial CS. 


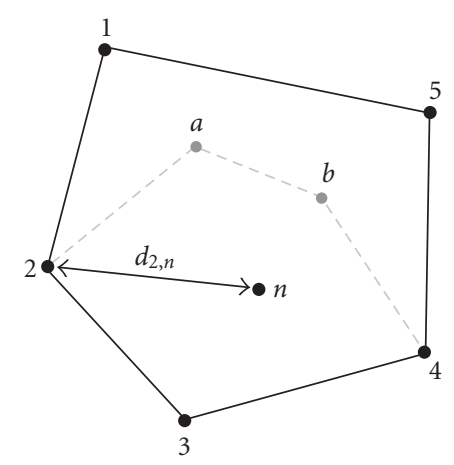

FIGURE 2: In this example, node $n$ is surrounded by nodes $1, \ldots, 5$ which form the convex hull of neighbors. $a$ and $b$ are called inner nodes, which will be ignored at the moment. The location of $n$ has yet to be determined with the help of the known locations of $1, \ldots, 5$ and their distances $d_{k, n}$ to node $n$.

This process is repeated by all nodes. The rest of Čapkun's work deals with consolidating all local CS into a single global one.

\subsection{Triangulation}

The process of positioning (here within a plane) by means of either three known positions and three distances, or two positions and two angles is referred to in the literature as triangulation.

If three distances are known, circles are drawn around each of the three anchor points. The radius of each circle corresponds to the distance between the anchor point and the node to be positioned. The circles will yield a common intersection at the position of the floating node. For in-depth coverage, please refer to [6].

\section{PRECISION-AWARE CONVEX COMBINATION}

\subsection{Introduction to the weighting scheme}

We will now address our precision-aware position calculation, which assumes the following conditions. We consider an unlocalized node $n$ somewhere in an outdoor space that has a set of localized neighbors $k \in 1, \ldots, K$. A node $k$ is considered to be a neighbor of $n$ if both are within the same radio range and $k$ knows its own global position. Furthermore, we assume that all neighboring nodes enclose a convex patch around $n$. Figure 2 shows an example of such a setting. The convex patch will be denoted as the neighborhood patch. The aim of our approach is to derive the location $p_{n}$ of the new node $n$ from the (surrounding) neighbors.

\section{Hint}

In case $n$ is not contained in a neighborhood patch, the proposed convex combination is not possible. This can happen if the node is part of the convex hull of the entire network, as is true, for example, for node 2 in Figure 2, and there is another case in which a convex combination will not be possible. In a very sparsely scattered network, inner nodes may be connected in one direction only. This would theoretically be the case if node $b$ was connected to nodes " 1 ," " 2 ," and " $a$ " only. Note that this does not mean that the node in question cannot be positioned by triangulation if it has at least three neighbors. It only means that in these special cases, the proposed convex combination cannot be applied.

Except for the special case mentioned above, two neighboring nodes on the convex hull $i, j \in\{1, \ldots, K\}$ in different places will suffice to localize node $n$ if the distance between $i$ and $n$, denoted as $d_{i, n}$, and the distance $d_{j, n}$ is known. As described in Section 2.3, the two circles around $i$ and $j$ are chosen such that they intersect at node $n$. They will then produce two intersections. For the following reason, in our case, only two nodes suffice to determine a node's position. In the proposed convex combination, only neighboring nodes on the convex hull are involved in estimating the position of node $n$. As a consequence, one intersection (the valid one) will be inside the patch, while the invalid one lies outside. Thus, it can be excluded in the first place. Another simple way to identify the right choice for the position of $n$ is to look for a cluster of suggested positions. Only those positions near the cluster center are valid in our context; the alternative positions are scattered around the patch and are not considered. The cluster will be obtained anyway in the subsequent calculation.

\subsection{What is the gain of unequal weighting?}

As stated above, only two known locations $p_{i}$ and $p_{j}$ along with the distances to nodes $n, d_{i, n}$, and $d_{j, n}$ are needed to derive the location of node $n$. However, we have to keep in mind that all distance measures will be more or less disturbed. Obviously, more accurate results can be expected by incorporating all neighbors rather than relying only on two of them. We can imagine a situation in which all nodes "provide a suggestion" for the estimated location $p_{n}$ of node $n$. Some suggestions by nodes within a close proximity will be more accurate, while others will be less reliable due to long distances. So ideally, the position estimate from a near-by node should contribute more to the calculation of $p_{n}$ than the position suggested by those nodes with weak incoming signals (the perception of the signal strength is always based on the perspective of node $n$ ).

Our approach expresses the location $p_{n}$ by means of a convex combination $[7,8]$ of the position suggestions $p_{n}^{(k)}$ coming from each neighbor $k$, as shown in (2). We denote the suggestion of node $k$ as $(k)$ in the superscript. The $n$ in the subscript represents the suggested position of node $n$

$$
p_{n}=\sum_{k=1}^{K} l_{k} p_{n}^{(k)}, \quad \sum_{k=1}^{K} l_{k}=1 .
$$

The weights $l_{k}$ that define the influence of each position $p_{n}^{(k)}$ should satisfy the following constraint. As node $n$ approaches an arbitrary node $k$, there should be an increasing contribution of location $p_{n}^{(k)}$ to $p_{n}$. Generally, the contribution of the location $p_{n}^{(k)}$ to $p_{n}$ should be inversely proportional to the distance between $n$ and $k$, causing nearby nodes to have more influence on each other than distant nodes will 


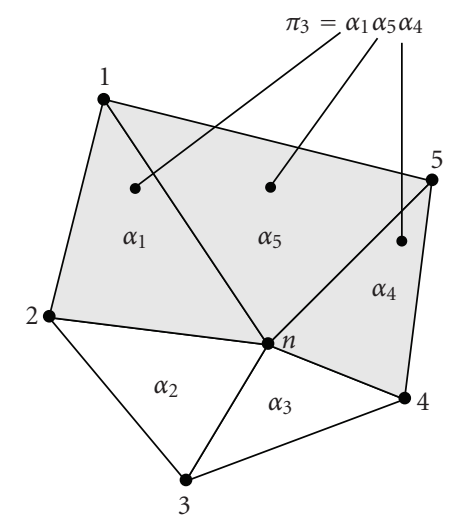

FIgURE 3: An area $\alpha_{k}$ is formed by the inner node $n$ and two neighboring outer nodes of the convex polygon. The product $\pi_{3}$ can be thought of as the weighting factor of node 3 . Note that $\pi_{3}$ consists of the dark-shaded areas which do not share the common edge between node $n$ and node 3 .

have. Intuitively speaking, we might say that if $k$ is close to $n$ it will have a better understanding of position of $n$ and should thus be given a greater weight.

Before going into detail on how to obtain the weights for each node, we will introduce the atoms from which they are constructed, namely the areas $\alpha_{k}$. Figure 3 shows that each area $\alpha_{k}$ is formed by the triangle $\Delta\left(p_{n} p_{k}, p_{(k \bmod K)+1}\right)$. The slightly clumsy expression in the subscript $(k \bmod K)+1$ of the last vertex of the triangle is required to calculate the area of $\alpha_{K}$. For $\alpha_{K}$, the positions of both the last and of the first nodes are needed, which are obtained by using the modulo operator. The calculation or area $\alpha_{k}$ is done according to Heron's formula [9]:

$$
\begin{aligned}
s & =d_{k, k+1}+d_{n, k}+d_{n, k+1}, \\
\alpha_{k} & =\frac{\sqrt{s\left(s-2 d_{k, k+1}\right)\left(s-2 d_{n, k}\right)\left(s-2 d_{n, k+1}\right)}}{4} .
\end{aligned}
$$

Since the location $p_{n}$ is not yet known, the areas $\alpha_{k}$ have to be obtained using the distances between the nodes. This is done in (2) by means of the distances $d_{i, j}$ between two nodes $i$ and $j$ :

$$
\pi_{k}=\frac{\prod_{i=1}^{K} \alpha_{i}}{\alpha_{k-1} \alpha_{k}}=\alpha_{1} \alpha_{2} \cdots \alpha_{k-2} \alpha_{k+1} \cdots \alpha_{K} .
$$

Equation (4) almost immediately derives the weights for each sensor node (or more precisely for the location suggested by each of them) from the weight $\pi_{k}$ in (4). The only difference between the weights $\pi_{k}$ and the actual weights $l_{k}$ that are used for the convex combination in (2) (and which so far have been omitted for didactic reasons) is that the $l_{k}$ will be normalized in order to sum up to 1 , which is not the case for the sum over all $\pi_{k}$.

The calculation of $\pi_{k}$ is now described more intuitively. To obtain the (unnormalized) weight $\pi_{k}$, multiply all areas except for the two areas which share the common edge between node $n$ and $k$. Figure 3 makes clear that the two triangles $\alpha_{2}$ and $\alpha_{3}$ are not included in the product. Now it be- comes obvious why the influence of a particular node $k$ increases as it is approached by $n$. The reason is that, as shown in Figure 4, the two white areas $\alpha_{2}$ and $\alpha_{3}$ become smaller and smaller, while the other three dark areas tend to cover more of the entire polygon. However, the weight $\pi_{3}$ does not include the shrinking areas but only the dark-shaded growing areas. All the other weights $\pi_{k \neq 3}$ actually do include the shrinking areas in their product. Note that all weights $\pi_{k \neq 3}$ except for $\pi_{3}$ contain a white area (resp., a small factor) in their product. Multiplying by a small factor $\alpha$ also renders the according weights $\pi_{k \neq 3}$ small, while $\pi_{3}$ is not influenced by the two shrinking triangles.

Finally, (4) defines the actual weights $l_{k}$ for the convex combination in (2), which eventually leads to an optimized approximation of the true location of $n$. The $l_{k}$ are simply a version of the $\pi_{k}$ normalized in order to sum up to 1 :

$$
l_{k}=\frac{\pi_{k}}{\sum_{i=0}^{K} \pi_{i}} .
$$

We did not yet define how to obtain what we have so far called node $k$ 's "suggestion" $p_{n}^{(k)}$ for the location of node $n$. At this point, the reader should not be misled into believing that a convex combination of the actual locations of the neighboring nodes may lead to the true location of $n$ as shown in (5):

$$
p_{n}=\sum_{k=1}^{K} l_{k} p_{k}+\operatorname{error}\left(p_{n}\right)
$$

In fact, a simple convex combination of the known world coordinates $p_{k}$ of the neighboring nodes would also result in an approximation of the location $p_{n}$. Figure 5 displays the magnitude of the displacement error that would be made using this particular kind of simplified approximation. Areas near the vertices of the polygon suffer more than those in the middle from smaller deviations from the actual coordinates. It can be shown that the error made using (5) is zero over the whole polygon only in the special case of equilateral neighborhood polygons.

Obviously, the combination of the neighbors' coordinates $p_{k}$ has to be replaced by using what was already mentioned as "node $k$ 's suggestion" $p_{n}^{(k)}$ of the assumed location $p_{n}$, which is again used in (6):

$$
p_{n}=\sum_{k=1}^{K} l_{k} p_{n}^{(k)}
$$

Geometrically, $p_{n}^{(k)}$ is obtained by drawing a circle around node $k$, as sketched in Figure 6. Only locations at the boundary of the circle are candidates for location $p_{n}^{(k)}$. Intersecting with another circle around node $k-1$ or $k+1$ provides a first estimate. However, there is no need to choose either $k-1$ or $k+1$ because both of them can be utilized at the same time. As a result, two valid intersections $s_{1}$ and $s_{2}$ evolve. The midpoint $p_{n}^{(k)}$ on the line between both intersections can be used as an improved approximation of the location of $n$. Note that the distance $d_{k, n}$ between a node $k$ and a node $n$ can only be estimated and is thus error-prone. We denote the approximation of $d$ as $\tilde{d}$. If the estimates were correct, $s_{1}$ and $s_{2}$ would 


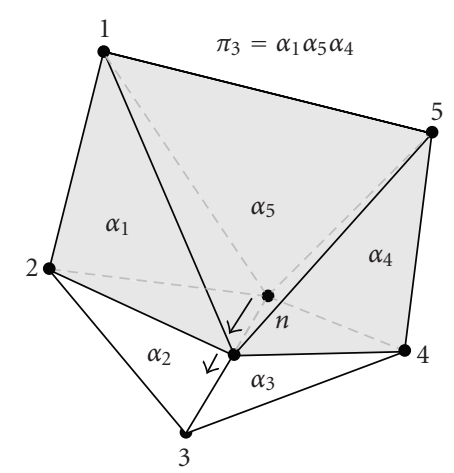

Figure 4: As node $n$ approaches node 3 , the dark areas $\alpha_{1}, \alpha_{5}$, and $\alpha_{4}$ gain area at the expense of the white areas $\alpha_{2}$ and $\alpha_{3}$. Since $\pi_{3}$ is composed only of the dark areas, its overall influence converges against that of the entire polygon area.

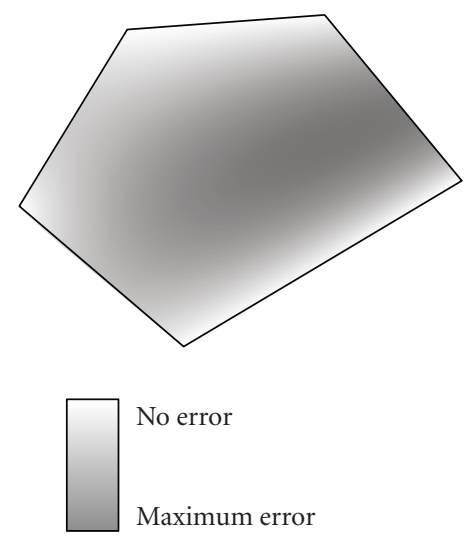

FIGURE 5: Simply combining the known global location of the neighboring nodes to estimate the coordinates of node $n$ results in an error of varying magnitude depending on the true location of $n$.

meet exactly at $p_{n}$, so that two intersections would not be necessary.

We will show in the next section that this weighted combination causes a smaller average mistake than does an equally weighted sum of all $p_{n}^{(k)}$.

\section{EVALUATION OF THE PRECISION-GAIN}

Let us quickly summarize what we did in the last section and analyze the benefits of our approach. A sensor node in an unknown place has to derive its position with the help of surrounding nodes which already know their global coordinates. Each of them can estimate the distance to the unlocalized inner node with a degree of uncertainty. By means of triangulation, a first guess of a position can be made with the help of two nodes.

In a straightforward procedure, the estimates of surrounding pairs of nodes could be averaged. If we interpret a single position guess as a random variable with a specific standard deviation $\sigma$, then the average out of $n$ samples will decrease $\sigma$ by the factor of $\sqrt{n}$.

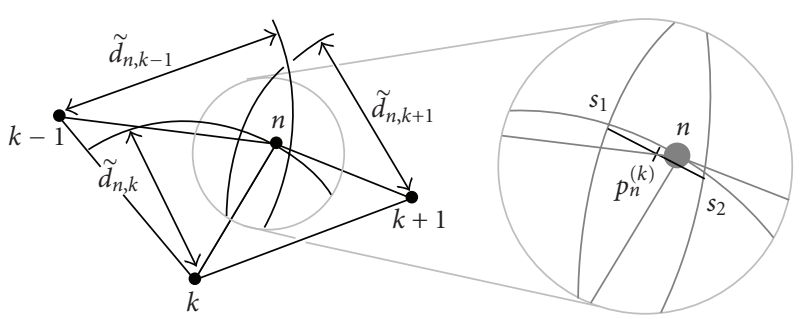

FIGURE 6: Location of node $n p_{n}^{(k)}$ is calculated from the viewpoint of node $k$. A circle around $k$ is intersected by circles around $k-1$ and $k+1$. The two arising valid intersections are denoted as $s_{1}$ and $s_{2}$. The location right between those two intersections can be used as the approximation $p_{n}^{(k)}$ for the location of $n$ proposed by node $k$.

In contrast to the equally weighted average, the unequal weighting scheme aims to improve the precision further by moving the weights to those nodes which are able to make a better guess. In more statistical terms, we focus on the contributing nodes with a lower variance while still maintaining the benefits of the mean calculation consisting of several measures. We exploit the fact that an estimate for a distance to a distant node tends to be coarser than the guess of a node in a close proximity.

In this section, we want to analyze under which conditions the unequal weighting scheme improves the precision gain the most by means of a simulation. Before we go into detail, we examine in the following subsection under which circumstances our assumption holds true that estimates of long distances are coarser as compared to short distances.

\subsection{Background on distance estimates}

Most positioning approaches need estimates of distances between nodes. Depending on the capabilities of the hardware, distances can be derived from the strength of the incoming radio signal, which is often referred to as Received signal strength indicator (RSSI), for example, in the context of the RADAR system [10]. As an alternative, the time of arrival (TOA) or time difference of arrival (TDOA) of beacons between communicating nodes can be used to estimate mutual distances.

In order to evaluate the fitness of a typical sensor node platform, we did some tests on TDOA with radio beacons on the ESB nodes. However, TDOA did not prove to be a feasible approach in this context. The main reason is the coarseness of the internal clock. The Texas Instruments MSP430 processor used for the ESB nodes is clocked at about $8 \mathrm{MHz}$. Even if an incoming bit (which is the shortest unit the transceiver can send) could be detected within a single cycle of the processor, it would take $1.25 \times 10^{-7}$ seconds. If the signal travels at about $300000 \mathrm{~km} / \mathrm{s}$, a radio beacon will bridge a distance of about 37.5 meters within one clock cycle. In practice, the detection of a bit takes much longer on most sensor node platforms since they are not optimized for the fast detection of radio signals. The order of magnitude of the error and the error variance introduced by these delays renders TDOA-based 
approaches unsuitable for most of today's sensor node platforms.

Theoretically, sound beacons are more suitable for TDOA-based positioning on sensor nodes as sound travels only about $300 \mathrm{~m} / \mathrm{s}$. Early work on range estimation using acoustic sensing has been done by Girod and Estrin using standard PCs and Linux [11]. Sallai et al. report an average error of below $10 \mathrm{~cm}$ using MICA nodes [12].

On the ESB nodes, a single clock cycle allows sound to travel only about $0.0375 \mathrm{~mm}$. So even if the detection of the beacon takes 1000 cycles or more, the accuracy is still sufficient. In our evaluations, the detection of the specific frequency the piezo-buzzer could produce proved to be challenging. Particularly for longer distances, the microphone was not sensitive enough to ensure a reliable detection. Furthermore, the 2048 bytes of RAM do not encourage the implementation of sophisticated signal analysis algorithms, especially since the memory is shared with the operating system.

Acoustic distance measurements have also been done by Sallai et al. [12]. The authors encountered a tight correlation between the sound propagation delay and internode distances, however, with larger outliers for increased distances. According to their analysis, these outliers are due, for example, to reflections or multipath propagation. These phenomena tend to become more important with increasing distance, while short distances often allow the signal propagation along the direct line of sight. So even for TDOA-based approaches, we can conclude that longer distances tend to introduce larger errors.

As a consequence of the simplicity of our hardware used, we tried to focus on the received signal strength. Its simplicity makes it suitable for very simple devices with no audio sensors and actuators, and even for very constrained resources of even simpler hardware often referred to as smart dust. The readings of the signal strength allow only for very coarse distance estimates, which was the main motivation to come up with our approach.

As we assume that the hardware has to be used as provided by the sensor node, we focused on its particular characteristic to find a way to improve the precision of the distance estimate. It proved to be helpful to have a closer look at the received radio signal strength $s$ between two nodes, which is known to degrade inversely proportional to the squared internode distance $d$ :

$$
\mathcal{s} \sim \frac{1}{d^{2}}
$$

A sensor node samples the incoming signal in equally sized quantization steps. This means that the resolution of the received radio signal in the proximity of a node is relatively high. The greater the distance between two nodes, the larger the interval that has to be covered by a single digital value. Another more practical cause of error influence is that the detection of bits, for example, by means of a rising and falling radio signal, becomes more error-prone over larger distances on average.

For the sake of completeness we want to mention another class of positioning algorithms which do not require distance
FLOAT improvement(position $\mathrm{P}$, nodes $[\mathrm{k}] \mathrm{N}$ )

$/ /$ calculate distances to all spanning nodes

FOR EACH of the $\mathrm{k}$ nodes $\mathrm{N}[\mathrm{i}]$ spanning the patch BEGIN

dist_PN $[\mathrm{i}]=$ distance $(\mathrm{P}, \mathrm{N}[\mathrm{i}])$

dist_PN $[\mathrm{i}]+=$ error(dist_PN $[\mathrm{i}]) \quad / /$ and distrb

by certain error

END

// each triple $(\mathrm{i}, \mathrm{i}+1, \mathrm{i}+2)$ of nodes

// creates a position estimate

FOR EACH of the $\mathrm{k}$ nodes $\mathrm{N}[\mathrm{i}]$ spanning the patch position_suggestion $[\mathrm{i}]=$ triangulate(dist_PN[i], dist_PN $[(\mathrm{i}+1) \mathrm{MOD} k]$, dist_PN $[(i+2)$ MOD k]

// the final position is a weighted sum

// of the position estimates

estimation_weighted $=$ estimation_trivial $=0$

FOR EACH of the $\mathrm{k}$ nodes $\mathrm{N}[\mathrm{i}]$ spanning the patch BEGIN

estimation_weighted $+=$ position_suggestion[i]

* weight [i]

estimation_trivial += position_suggestion $[\mathrm{i}] / \mathrm{k}$ END

improvement $=\mathrm{ABS}(\mathrm{P}$-estimationtrivial $)-\mathrm{ABS}(\mathrm{P}-$

estimation_weighted)

return improvement

Algorithm 1

estimates. They operate based only on the mere connectivity between nodes. APIT is an instance of these range-free localization schemes which has been proposed by He et al. It operates based on beacons that allow a node to determine whether it is inside or outside a particular triangle [13]. By intersecting several triangles, the valid area can be narrowed down successively. Doherty et al. have proposed a convex position estimation which is also based on simple connectivity constraints [14]. The authors analyze the precision of position estimates in a more or less populated neighborhood. To take the remaining uncertainty of the estimates into account, they define bounding boxes for candidate regions.

Niculescu and Nath suggested to use the angle of arrival $(A O A)$ of a signal to determine the position by means of triangulation. Here, the problem of determining mutual distances is shifted to determining the direction of a sender [7].

\subsection{Assessment of the unequal weighting scheme}

In order to simulate other topologies of nodes or error characteristics than the ones proposed below, our simulator can be obtained free of charge. (The tar-archive of the simulation can be obtained at http://www.informatik.uni-mannheim .de/ haensel/sensor_location.tgz.). 
TABLE 1: The column example topology shows four example topologies in which the positioned nodes span a patch. Column normal average shows the mean deviation between the true position and the calculated one for the average computation in absolute distance units. Unequal weights column depicts the same calculations based on unequal weights. The mean improvement over the equally weighted average is provided by column gain.

\begin{tabular}{|c|c|c|c|c|c|c|c|}
\hline Example topology & Normal average & Unequal weights & Gain & Example topology & Normal average & Unequal weights & Gain \\
\hline Triangle & 27.4 & 18.7 & $31.8 \%$ & Quadrangle & 25.3 & 19.0 & $24.9 \%$ \\
\hline 5 vertices & 24.8 & 19.5 & $21.4 \%$ & Colinear & 26.2 & 23.2 & $11.5 \%$ \\
\hline
\end{tabular}

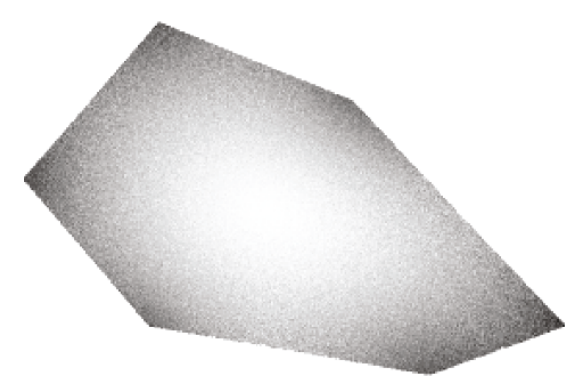

FIGURE 7: The precision gain of the unequal weighting scheme over the normal average is displayed for an example topology spanned by six positioned nodes. The strongest improvements displayed in dark shades take place at the edges and vertices of the patch. The improvements vanish towards the center of gravity in the middle because here the weights become more and more equal, thus converging against an equally weighted average.

In the simulation, the neighborhood patch is built up by means of the spanning nodes. Then, the software iterates over each inner position $\mathrm{P}$ on a fine-grained grid. For a given position $\mathrm{P}$, the pseudo code below evaluates the gain between our proposed unequal weighting scheme and an equally weighted one. In particular, the variable estimation weighted contains the error caused by the unequal weighting scheme, while estimation $_{\text {trivial }}$ contains the error of the equally weighted average. Both error values state the spatial distance between the actual position $\mathrm{P}$ and the calculated one. A difference of zero means that the exact position was calculated. Any other positive value reflects the magnitude of the distance vector between $\mathrm{P}$ and the assumed position.

In the end, the proposed approach is an improvement only if its calculated position is closer to the real position $\mathrm{P}$ as compared to the equally weighted scheme. Figure 7 shows a plot of the positioning improvement return by Algorithm 1 .

If the distances dist ${ }_{P, N}$ were all precise, the calculated coordinate of $\mathrm{P}$ should match the true one, perfectly. However, in a real-world scenario, those distances can only be guessed at, as we have pointed out in Section 4.1. Depending on the internode distance, each distance measurement is disturbed by a certain error in the simulation.

The degree of distortion of a measurement and the improvements achieved in the end are put as a fraction of the radio range below.

The error (error(dist) in the pseudocode) added to the internode distances never drops below $5 \%$ of the radio range for short distances, and rises to up to $20 \%$ for distances up to the maximum radio range. As stated in Section 4.1, the assumption is that longer distances are more prone to exhibit larger errors than are shorter ones.

Figure 7 shows a plot of the improvement over a sample patch in which darker areas indicate stronger improvements and lighter areas less improvement. Obviously, the vertices and the boundary of the patch profit more from the unequal weighting scheme, while the inner part of the patch exhibits no significant gain. The reason for this is that the positions in the middle are about equally distant from the spanning nodes. As a consequence, their weights are also more or less equal. So the positioning quality between the equally and unequally weighted scheme can only be marginal, the more a position $\mathrm{P}$ converges into the center of gravity of the patch.

Table 1 and the accompanying Figure 8 provide a closer quantitative analysis of the precision gain over the different patch topologies.

Figure 8 shows different configurations of nodes, a triangle, a rectangular quadrangle, and an irregular shape with five vertices. Again, the darker shades show regions in which larger improvements can be achieved, while the light shades depict areas with little or no improvement. All images including Figure 7 exhibit a noisy salt-and-pepper-like structure, in particular in the darker areas. This shows that positioning improvements occur irregularly. In other words, some positions profit highly from the unequal weighting, while others are hardly or not at all influenced.

Responsible for this phenomenon is the introduced error. As described above, the inter-node distances are disturbed as much as $20 \%$ of the node's radio range. However, the equally distributed random error can by coincidence cause both strong disturbances or none at all. So sometimes the distortion of the inter-node distances will by chance be close to zero. If the error is small or negligible, then the difference between the results of the equal and the unequal weighting schemes can also only be marginal. Because all the summands of which the weighted average consists are good estimates. And moving the weight from one good estimate to another hardly alters the final result. As a consequence, the precision gain will be negligible as well.

For a more intuitive example, the reader may imagine the bullet holes caused by a shotgun. Some will be strong outliers, while others may be clustered close to the target point. Calculating the target point based on those which are closely scattered around the actual target will result in a good approximation, which is what the weighted average tries to achieve. However, including all outliers with a high variance will result in a higher variance of the average as well, thus yielding worse results. However, if by chance there are no 


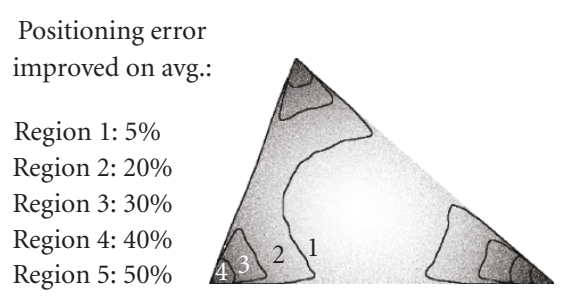

(a) Three nodes span a triangular patch

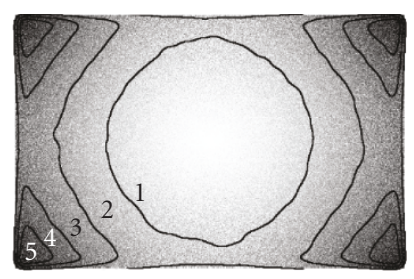

(b) Rectangular quadrangle

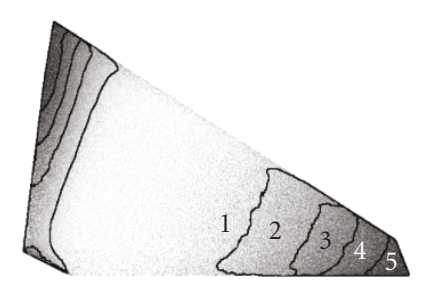

(c) Five vertices of which four nodes form two pairs

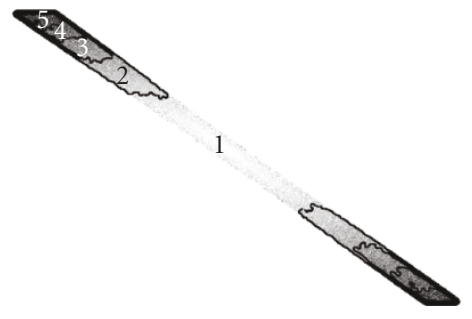

(d) Four almost colinearly positioned nodes spanning a slim patch

FIGURE 8: Different topologies of nodes were evaluated to assess their positioning gain. Numerical figures are given in Table 1. Obviously, the largest improvements which cut down the error about 50\% on average are made at positions which have neighbors (the vertices of the polygon) near by and in a larger distance. Unequal positioning achieves the least improvements if all nodes have about the same distance (in the middle of the polygons).

strong outliers, weighting some bullet holes unequally will not change the average position significantly.

Table 1 shows the positioning gain. For the triangle, we can see that the deviation of the distance in absolute units is 27.4 for the equally weighted sum and 18.7 for the unequally weighted sum. This means that on average, an improvement of about $31.8 \%$ was achieved. For the other topologies, the error can be cut by $25 \%-21 \%$ which is a significant improvement, given that the scheme exploits the characteristics of the measurements only and does not alter the hardware at all.

Figure 8(d) shows another topology of four nodes that are almost co-linear. As a result, they form a long and narrow patch. In this simulation, the gain the unequal positioning can achieve is lower, at only about $11 \%$. This effect can be explained by the patch's geometry. As seen in the examples of Figure 8 , the outer regions of the patch profit most from the proposed scheme.

Let us consider a circular patch. If we increase the radius, the outer area increases proportional to the square of the radius. In contrast, a linear structure increases proportional only to its length. Though the slim patch has an area as well, it's growth does not result in a significant gain in the beginning.

Put into more mathematical terms: the function $f(x)=$ $x^{2}$ remains close to the abscissa for some time before gaining large values. For this reason, mostly colinear structures gain area only after significant scaling. So the outer parts of these patches which profit most from the proposed approach do not grow that easily, which results in smaller improvements since the ratio between the outer regions which profit much and the inner regions which profit least remains more equilibrate for some time. Even more formally we can simply state that $x \times x$ grows faster than $(x+c) \times(x-c)$.
The degenerated patch of Figure $8(\mathrm{~d})$ does not play an important role in practice since a set of randomly scattered nodes do not often produce these topologies.

\subsection{Computational requirements}

In the context of sensor networks, the feasibility and the efficiency of an algorithm have to be taken into account. The calculations we performed are composed of a sum of estimates $p_{n}^{(k)}$ with one weight $l_{k}$ for each summand (see (6)). Since the weights $l_{k}$ essentially are the triangular areas $\pi_{k}$, scaled in order to sum up to one, the total effort is increased only by a constant factor. Only the areas $\pi_{k}$ are each composed of all distance estimates. So $n$ neighbors lead to a computational effort proportional to $O\left(n^{2}\right)$. A number of about 10 neighbors lead to several hundred multiplications, additions, and a few root calculations. However, even the highly energy efficient MSP430 processor running at $8 \mathrm{MHz}$ can perform about 2.6 million instructions per second with an average number of three clock cycles per instruction. Since the architecture has zero wait states (the memory is in sync with the processor), this performance is actually achieved. After all, the calculations only have to be done once in the startup phase of the network, so the requirement for additional time and energy is negligible.

\section{CONCLUSION AND OUTLOOK}

We have proposed an approach to positioning a wireless sensor node by means of the distance from surrounding nodes with known world coordinates. We consider each localized node to suggest a new node's position. But not all suggestions will be of the same quality. The distant nodes 
will propose coordinates with greater uncertainty than will nearby nodes. The position of the unlocalized node is obtained by a weighting scheme which emphasizes coordinate suggestions of nearer nodes, thus gaining certainty over the new node's true position.

From the field tests with the ESB platform, we know that the degradation of signal quality is not influenced significantly by the internode distances up to a certain range, but drops relatively fast at a further distance. In our future work we will try to adapt the weighting scheme more closely to the reception characteristics in order to strengthen the influence of nodes within the more deterministic shorter rang.

\section{APPENDIX}

Our evaluation is based on a simulation, which we provide for download under the GPL at www.informatik.unimannheim.de/ haensel/location_simulation.tgz.

It runs under GNU/Linux using the simple direct media layer (SDL). The polygon can be chosen arbitrarily but has to be defined in the source code in the beginning.

\section{REFERENCES}

[1] U. E. Ruttimann, R. A. J. Groenhuis, and R. L. Webber, "Restoration of digital multiplane tomosynthesis by a constrained iteration method," IEEE Transactions on Medical Imaging, vol. 3, no. 3, pp. 141-148, 1984.

[2] N. Bulusu, J. Heidemann, and D. Estrin, "GPS-less low-cost outdoor localization for very small devices," IEEE Personal Communications, vol. 7, no. 5, pp. 28-34, 2000.

[3] T. Adebutu, L. Sacks, and I. Marshall, "Simple position estimation for wireless sensor networks," in London Communications Symposium (LCS '03), pp. 300-305, London, UK, September 2003.

[4] L. Doherty, K. S. J. Pister, and L. El Ghaoui, "Convex position estimation in wireless sensor networks," in Proceedings of the 20th Annual Joint Conference of the IEEE Computer and Communications Societies (INFOCOM '01), vol. 3, pp. 1655-1663, Anchorage, Alaska, USA,, April 2001.

[5] S. Capkun, M. Hamdi, and J. Hubaux, "GPS-free positioning in mobile ad-hoc networks," in Proceedings of the 34th Hawaii International Conference on System Sciences (HICSS '01), p. 255, Big Island, Hawaii, USA, January 2001.

[6] T. Haenselmann, An FDL'ed Textbook on Sensor Networks, chapter 3, 4, University of Mannheim, Mannheim, Germany, 2007.

[7] D. Niculescu and B. Nath, "Ad hoc positioning system (APS) using AOA," in Proceedings of the 22nd Annual Joint Conference of the IEEE Computer and Communications Societies (INFOCOM '03), pp. 1734-1743, San Francisco, Calif, USA, MarchApril 2003.

[8] C. T. Loop and T. D. DeRose, "Multisided generalization of Bezier surfaces," ACM Transactions on Graphics, vol. 8, no. 3, pp. 204-234, 1989.

[9] D. P. Robbins, "Areas of polygons inscribed in a circle," The American Mathematical Monthly, vol. 102, no. 6, pp. 523-530, 1995.

[10] D. Niculescu and B. Nath, "Dv based positioning in ad hoc networks," Telecommunication Systems, vol. 22, no. 1-4, pp. 267-280, 2003.
[11] L. Girod and D. Estrin, "Robust range estimation using acoustic and multimodal sensing," in Proceedings of IEEE International Conference on Intelligent Robots and Systems (IROS '01), vol. 3, pp. 1312-1320, Maui, Hawaii, USA, October 2001.

[12] J. Sallai, G. Balogh, M. Maróti, A. Lédeczi, and N. Kusy, "Acoustic ranging in resource constrained sensor networks," Tech. Rep. ISIS-04-504, Institute for Software Integrated Systems, Vanderbilt University, Nashville, Tenn, USA, 2004.

[13] T. He, C. Huang, T. Abdelzaher, B. M. Blum, and J. A. Stankovic, "Range-free localization schemes for large scale sensor networks," in Proceedings of the 9th Annual International Conference on Mobile Computing and Networking (MOBICOM '03), pp. 81-95, San Diego, Calif, USA, September 2003.

[14] L. Doherty, K. S. J. Pister, and L. El Ghaoui, "Convex position estimation in wireless sensor networks," in Proceedings of the 20th Annual Joint Conference of the IEEE Computer and Communications Societies (INFOCOM '01), vol. 3, pp. 1655-1663, Anchorage, Alaska, USA,, April 2001. 